\title{
Space-Use Analysis in the Design of Neighborhood
}

\section{Walking Paths}

\author{
Carolina Martins, Teresa Heitor and Ana Tomé \\ Department of Civil Engineering, Architecture and Georesource, Univerisity of Lisbon, Lisbon 1049-001, Portugal
}

\begin{abstract}
SUA (space-use analysis) is about techniques that objectively describe environments and relate this description to specific problems of use. The purpose of this paper is to explore a space-use analytical tool—POE (post-occupation evaluation)—regarding a neighborhood accessibility condition. Based on a site-specific approach, the main research question is focused on how far neighborhood walking paths are compatible with different user needs, guaranteeing their safety and autonomy, as well as easiness of perception and use, thus providing access to local services and resources they need for participation in the day-to-day activities of community life. This paper is organized in three parts: The first one introduces the main concepts and explains how to ensure it in a practical application in urban design; The second part presents the practical approach, referring to the used methodology, the specific case-study and reached results; The third one establishes correlations between the two approaches and discusses the main findings.
\end{abstract}

Key words: Post-occupation evaluation usability, accessibility, neighborhood.

\section{Introduction}

Built environment disciplines define accessibility as the ease or difficulty associated with reaching a place. This concept is concerned to both the practicability of the geographical territory and users' mobility [1]. Within the framework of universal design, accessibility can be defined as the ability of the built environment to allow any person, regardless of age, mobility, visual, auditory or mental ability to use it without escort and without having to make a psycho-physical effort for adaptation. This concept is concerned to users' autonomy, safety and easiness of perception [2].

It is argued that combining these two approaches, it is possible to define accessibility in the built environment as being related with usability and intelligibility, which are interrelated. Usability addresses the capacity of the built environment to respond to user's needs and experience while intelligibility $[3,4]$ means the easiness of perception

Corresponding author: Carolina Martins, Ph.D. student, research fields: architecture, heritage and accessibility. E-mail: mgm.carolina@gmail.com. and use, considering how space allows users the possibility to orientate, i.e., to have the subjective ability to know their own position within the urban environment both in general and in relation to the starting and arrival points.

\section{Case Study}

\subsection{The Context}

The selected residential neighborhood is located in a large-scale urban development in Lisbon of about 510 ha. It is part of the process of urban expansion planned by the municipality in 1960s and developed along the next two decades in response to the housing shortage affecting the city.

The master design was prepared at the mid of 1960s by the city council following the debate opened at the CIAM VIII in 1951 regarding the ways in which architectural modernism impacted on the planning process in the postwar period [5]. It was planned to integrate a total of 11,500 dwellings and 55,300 inhabitants, activity areas including schools, markets, civic centres and small industries. It was also an attempt to break the Athens' chart urban design 
principles and practices by abandoning the free compositions with the natural environment. The urban layout was to be seen as a single entity instead of an agglomeration of individual neighborhoods served by their own centers. The overall layout took the form of a deformed H-shape, which was adjusted to the site topography. Shopping and other central uses were located in the midpoint and the residential ones were placed along the axes, forming six housing clusters or neighborhoods with a strong visual image. Their spatial structure was based on the urban block diagram with a system of primary and secondary streets designed to minimize through traffic and partially enclosed external spaces for public use. A peripheral circulation system for car traffic connected the residential neighborhoods to the central point and articulated the whole area to the city center. The master plan was not entirely executed. Due to the economic and demographic context of the time and, in particular to the housing shortage affecting the city, priority was given to housing developments. Neither the central area was developed, nor was the peripheral circulation system completed. Housing and economic developments were not accompanied by all necessary social and physical infrastructures, including public transports, education, local shopping, community and recreation facilities. Public realm spaces were disregarded as well the walking paths and the green landscape [5].

Although only partly built to the original design, the efforts made by the design team to re-conceptualize the traditional morphology of the city urban fabric and their attempts to create socially responsive environment that might meet the needs of the populations were not well successful. Both the condition of inaccessibility to social life's center and mono-functionality of the neighborhoods are brought almost to an extreme, and this area became isolated and fragmented area within the city acquiring a sense of "ghetto" [6]. In 1990s, the city council initiated a rehabilitation action plan, having established a combination of interventions to make the area more attractive, including the development of central area and the links to the city center.

\subsection{The Residential Neighborhood}

The residential neighborhood was developed in the end of 1970s. Formally, the plan organization was based on a set of urban blocks repeated from large to small scales, laying out the exterior public spaces, buildings, corridors and apartments in a branching system of elements. The slope conditions and the built structure lead to an excessively fragmented space.

Blocks are grouped into three different configurations: a single 9-story point-access tower and two different types of 4 to 5 -story connected slabs, which are organized as gallery-access with commercial spaces in the ground floor. With each, building elements containing several flats per floor are connected to stair elements to create a form of segmented linear. These slab elements define a perimeter edge, interlock to define several large courtyards, and align along the main circulation axe that runs through the site. A continuous configuration of buildings is located in the centre of the site. It comprises successive ramification, which define a series of external courtyards and an internal enclosed pedestrian street. The courtyard areas are used for parking and some defined play areas. The internal street has reached high levels of misappropriation and environmental neglect. After its occupation in mid-1980s, a policy of placing only low-income families and migrants into the area created a scenario of little investment, and segregation from the city of Lisbon. Moreover, the area was never built in its entirety, and major changes even at construction altered the ways in which movement was conceived in the master plan. Residents faced multiple physical barriers to move within it and to reach the central area as well as the others residential neighborhoods. This has led to the development of a complementary 
network of informal paths. The rehabilitation action plan carried out in 1990s gave particular attention to the improvement of the local accessibility conditions through the implementation of an integrated walkable neighborhood strategy together with the upgrading of public realm spaces. This has involved the redesign of the residential walking paths network, including footpaths, pavements and sidewalks, lighting, street furniture, signage and safe road crossings. To increase usability for all users, some normative measures were also applied concerning universal design principles. Although the improvement of the quality of footpaths, some of the formal paths are still not used by the residents in their daily activities in favor of the informal ones even when confronted with an infra-structured and more comfortable sidewalk next to them.

The analysis was focused on the formal and informal walking paths network linking the residential neighborhood to the shopping mall. Although not built according to pre-established design rules they are continuously and intentionally used in their daily routines. It was observed that formal paths are in general less used than the informal ones.

\section{Methodological Approach}

The methodological approach makes use of POE (post occupancy evaluation). POE is defined as the process of systematically evaluating the extent to which a facility, once occupied for a period of time, meets the intended organizational goals and user-occupant needs [7]. These methods aim to study the effectiveness of built environments from human user perspective [8]. The information from POE can be used not only to identify problems as well as to inform future projects [7].

POE techniques were applied to assess residents' satisfaction regarding the usability of the neighborhood walking paths network, i.e., how well their needs are met [9].

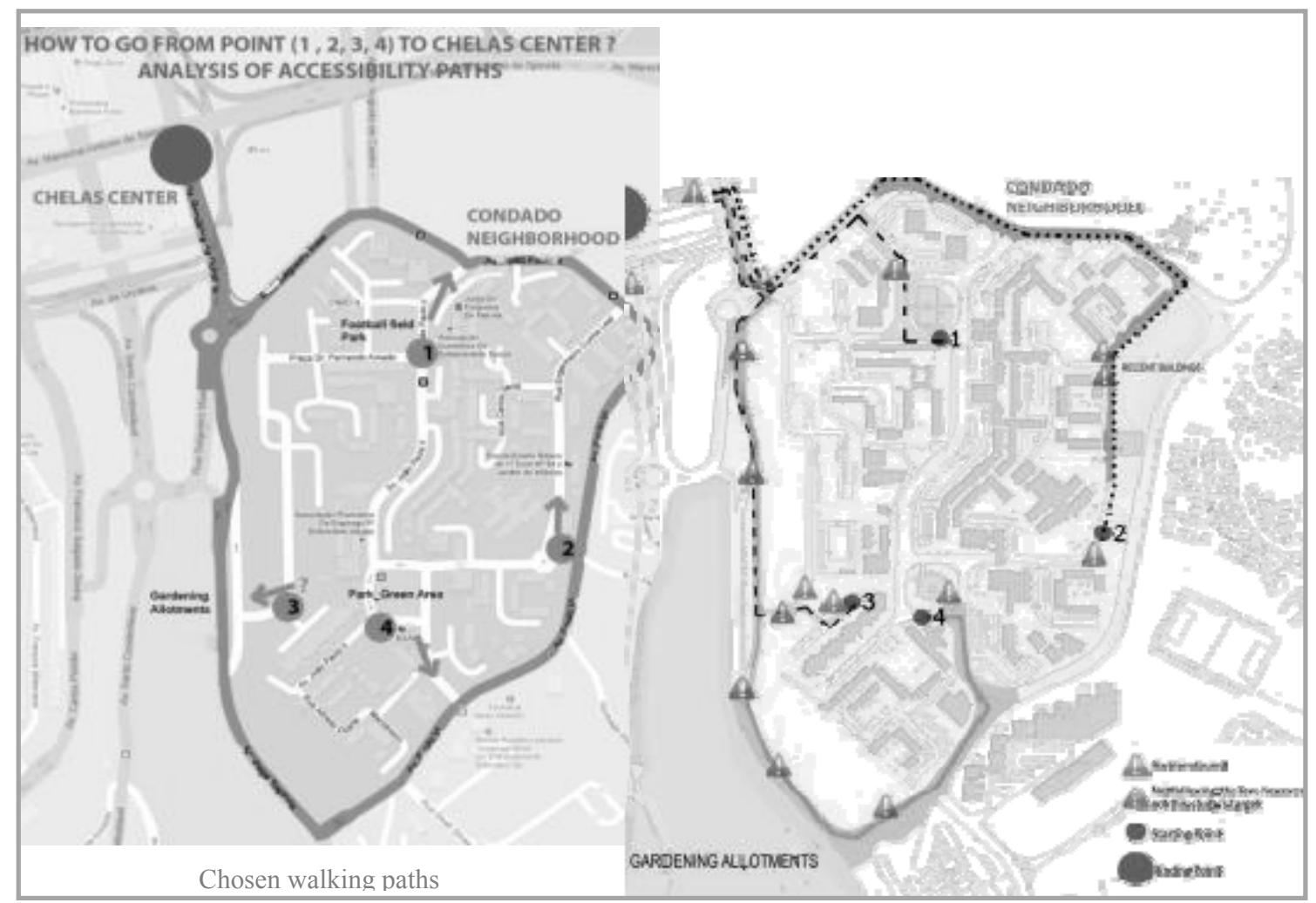

Fig. 1 Left: Starting from point 1/2/3/4 and finishing in Chelas Center it was possible to identify several problems-black points (Section 4); Right: example of black points sheet identifying access problems along the four paths. 


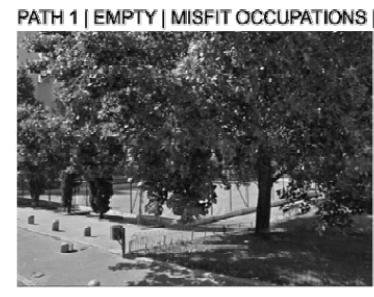

Starting Point -Public Football Field (See Fig. 3 for conclusions)

Visited and studied on a weekday Monday. Aiding by wheelchair's user (Feminine; 55-60 years old) + informal interview

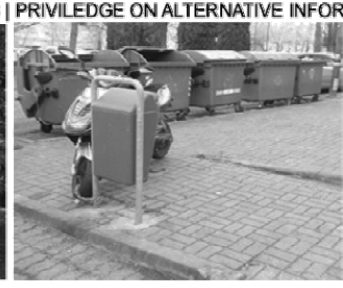

There's a correctly dimensioned ramp, but used for other purposes. Resident said: "There's no one there"

(a)

The reason might be lack of crosswalks to this point, no safety $=$ no use

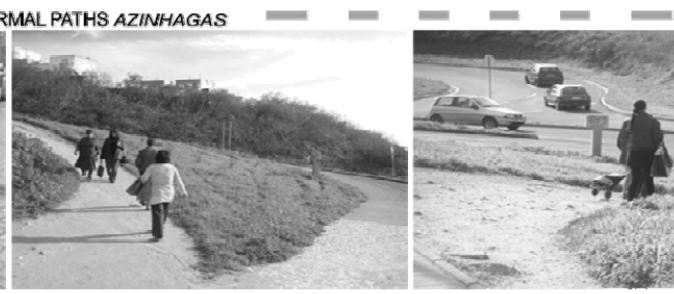

Then, almost in the destination point there's possible to find a question on the population's behavior. There's a sidewalk on the right and an informal path on the left, presenting the first one a weaker inclination, better dimensions, and accomplishing regulations of accessibility. In each 20 people, 1 passed in the programmed sidewalk and 19 passed in the improvised one. The informal path is shorter and older. The infra-structured one doesn't accomplish people's needs of faster and shorter accesses, being just following the road

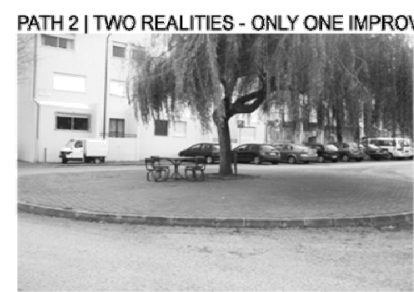

Starting Point - Roundabout

This path has two different realities - the old buildings of $J$ Area, and in the other

side the new buildings. With this last ones, Here, population claims the right to correct by their own, resulting in a possible accessible ramp, even this path became one of the most improved, specially in the "new side".
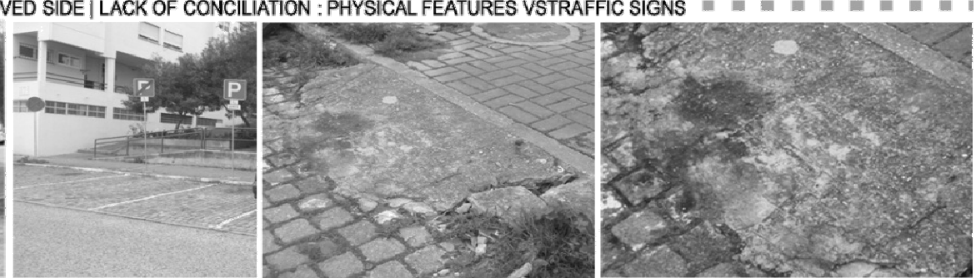

A situation of lack of conciliation of traffic signs with physical features. In this situation it's obvious the presence of parking for people with disabilities, however, what's worth for if then, there's no changes in the sidewalk? drawing the sign in blue, but althought it works it's not following the rules, and might not be safe.

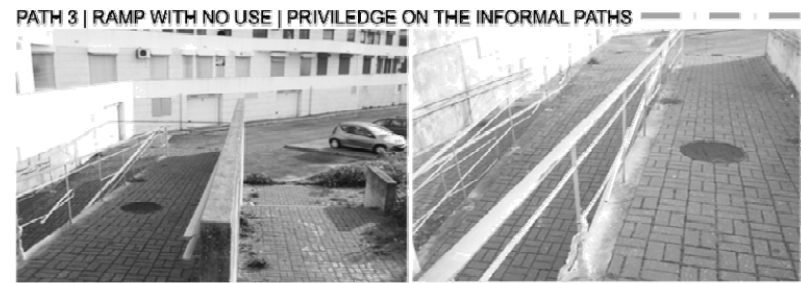

Starting Point - Ground floor of a chosen building for its isolation comparing to the other buildings. (Weckday - Tuesday). First impression: There's a ramp conecting 3 levels - upper level ( $\mathrm{J}$ area), ramp's level, lower level (parking). But then, it has no inclusive accesses from the upper level (stairs), neither to the lower level as there's no sidewalks to the parking area. So how can it have usability if then, it's no possible to reach by highly diverse group of people (wheelchairs for example)?

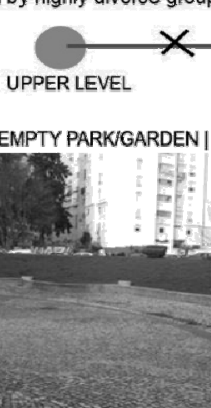

Starting Point - Park. It has green spaces, accessible path but it was empty (Observed during 3 hours/afternoon, 2 times). The problem might lie in the social dimension. The park is easily observed from outside

= vulnerable, easily surrounded In a small talk a woman complained: "I co cross it, Also no attractive points (shops) run. I have to."
One of the most important activities is their gardening allotments, for psycological and economical reesons. There exists improved sidewalks, accessible. However, people get here but then, there's no crosswalks to the other side to their gardening activities, becoming a dangerous path.

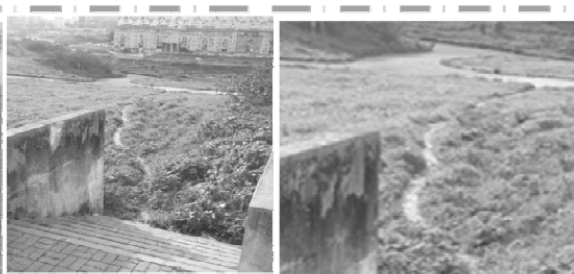

Contrary, t's possible to realise there's an improvised path next to the ramp, proving the ramp has absolutly no meaning in that context, although it is well dimensioned. Talking with 2 random people (women; both more then 60 years old) in the upper street, there were comments:

"Faster for that short-cut"; "A wheelchairs'user don't even get here, at least alone, so what's the problem of making this short-cut?"

\section{Fig. 2 Results on usability test.}

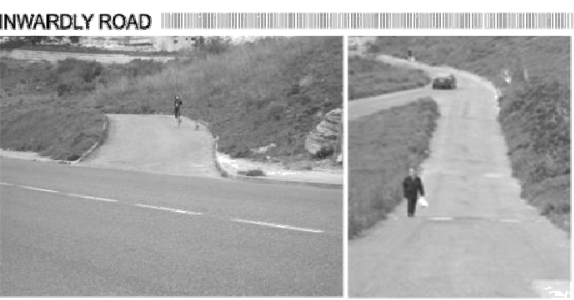

Going further to the supermarket there's another problem. The wider and nicer sidewalks mentioned before desappeared. The pedestrian path is interrupted by a road (again, a huge priviledge in the car) wth a very small inflow every day (according to residents), so why it exists?

That wide road inwardly to the main one has, actually, usability but for pedestrian mobility. People uses it as a sidewalk, as an important pedestrian route to the supermarket. 


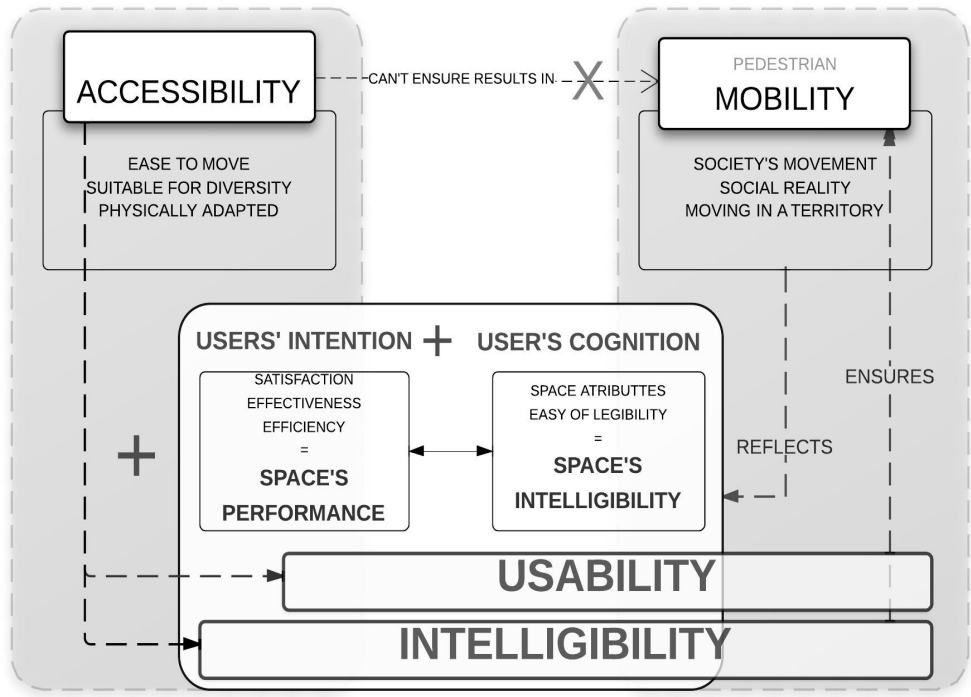

Fig. 3 Contributions of usability and intelligibility when designing relevant inclusive paths.

This study was based in the evaluation of the neighborhood walking paths network performance regarding residents' needs. It included: (1) the analysis of barrier-free standards and generic design guidance governing inclusivity to the built environment; (2) the inquiry of residents' daily routines, i.e., how far they use informal or formal paths. This has involved conventional measured survey, i.e., an inquiry procedure combining direct space-use observations, informal interviews with residents in order to obtain their perspective towards the paths performance and how well their needs are met, and photographic survey of relevant situations.

\section{POE-Usability Test}

The POE analysis was focused into four walking paths. Fig. 1 shows the situations where black points were found. These "black points" include: (1) empty paths, disregarding residents' needs and with/without obvious preference for informal paths or (2) paths that are physically accessible but population use it with different objectives.

It was observed that people privileged mostly the informal paths even when exist an infra-structured sidewalk next to it. This is a constant along the neighborhood, even in slope paths. Paths 1, 2 and 3

(Fig. 2) illustrate it. Also it was observed that although exists well-dimensioned sidewalks next to main roads, it is not possible to cross to the other side, making the path dangerous (Path 4). These sidewalks do not serve the neighborhood population. Since they lack crosswalks and traffic signs, they become irrelevant. Moreover, there are several ramps (Path 3) - that serve two inaccessible places.

\section{Conclusions}

This paper explored the practical role of usability in the evaluation of neighborhood walking paths. Usability is a key factor in providing neighborhood walking paths solutions that are compatible with different user's needs, guaranteeing their safety and autonomy, as well as providing easiness of perception and use.

The analysis strong suggests that people prefer informal paths for the fact they are easier to perceive and faster to achieve destination, ending up being deliberated. Plus, it is possible to conclude people designed those informal paths to have easy and faster access to the surroundings and at same time to reduce the neighborhood' isolation and fragmentation.

The proposed SUA tool is a relevant source for supporting design decisions. It not only offers the possibility to test different planning strategies and design proposals, as well as the outputs provided 
inform on the constraints and opportunities of neighborhood walking paths areas regarding user's satisfaction.

It is argued that to guarantee that a user-friendly neighborhood walking paths network there is a need to ensure people's intentions and user's cognition—social dimension (Fig. 3).

\section{References}

[1] A. Heylighen, M. Bianchin, How does inclusive design relate to good design?, Designing as a deliberative enterprise, Design Studies 34 (1) (2012) 93-110.

[2] J. Swain, S. French, C. Barnes, C. Thomas, Disabling Barriers-Enabling Environment, SAGE Publications Ltd., London, 2004.

[3] B. Hillier, J. Hanson, The Social Logic of Space, 2003rd ed., Cambridge University Press, Cambridge, 1984.

[4] J. Jacobs, The Death and Life of Great American Cities, Vintage Books, New York, 1992.

[5] T. Heitor, Spatial Vulnerability Spatial Vulnerability in the Design of Transition Spaces: A Comparative Study, in: R. Camstra, J. Smith (Eds.), Housing: Levels of Perspective, Amsterdam Study Centre for the Metropolitan Environment, Amsterdam, 1996, pp. 176-185.

[6] T. Heitor, From the Plan of Urbanization to the Current Situation: The (Mis) Configuration of the Public Space, Centro Português de Design, Lisbon 2004, pp. 151-155. (in Portuguese)

[7] W.F.E. Preiser, The evolution of post-occupancy evaluation: Toward building performance and universal design evaluation, in Federal facilities council technical report no. 145, Learning from our buildings: A state-of-the-practice summary of post-occupancy evaluation, National Academy Press, Washington, D.C., 2002.

[8] C.M. Zimring, J. Reizenstein, Post-occupancy evaluation: An overview, Journal of Environment and Behavior 12 (4) (1980) 429-450.

[9] S. Handy, Urban Form and Pedestrian Choices: A Study of Austin Neighborhoods, Transportation Research Record 1552, 1996. 